\title{
Platycodin D, a metabolite of Platycodin grandiflorum, inhibits highly metastatic MDA-MB-231 breast cancer growth in vitro and in vivo by targeting the MDM2 oncogene
}

\author{
YA KONG ${ }^{*}$, ZONG-LIANG LU*, JIA-JIA WANG, RUI ZHOU, JING GUO, JIE LIU, \\ HAI-LAN SUN, HE WANG, WEI SONG, JIAN YANG and HONG-XIA XU \\ Department of Nutrition, Daping Hospital and Research Institute of Surgery, \\ Third Military Medical University, Chongqing 400042, P.R. China
}

Received January 21, 2016; Accepted May 16, 2016

DOI: $10.3892 /$ or.2016.4935

\begin{abstract}
The objective of the present study was to explore the in vitro and in vivo anticancer effects of Platycodin D (PD), derived from Platycodin grandiflorum, on highly metastatic MDA-MB-231 breast cancer cells. Using the MTT assay, we found that PD inhibited MDA-MB-231 cell growth in a concentration-dependent manner, with an $\mathrm{IC}_{50}$ value of $7.77 \pm 1.86 \mu \mathrm{M}$. Further studies showed that PD had antiproliferative effects and induced cell cycle arrest in the G0/G1 phase. To explore the detailed mechanism(s) by which PD suppressed MDA-MB-231 cell growth, western blot analyses were used to detect the expression levels of proteins related to cell proliferation and survival. The data showed that PD decreased the expression of proteins related to the $\mathrm{G} 0 / \mathrm{G} 1$ phases, downregulated the protein expression of MDM2, MDMX, and mutant p53, and increased the expression levels of p21 and p27 in vitro. We verified the effects of PD on the expression of MDM2, MDMX, mutant p53, p21 and p27 using a pcDNA3-Flag-MDM2 plasmid and MDM2 siRNA transfection, and found that PD inhibited MDA-MB-231 cell viability by targeting MDM2 and mutant p53. Compared with the corresponding parental cells, the cells with siRNAMDM2 transfection had a greater decrease in cell viability and proliferation, while those with pcDNA3-MDM2 plasmid transfection did not show any increase in the effects of PD. We also established a MDA-MB-231 xenograft model in BALB/c nude mice, and found that PD significantly inhibited the growth of MDA-MB-231 xenograft tumors in these mice. The expression levels of various proteins in the tumor tissue
\end{abstract}

Correspondence to: Professor Hong-Xia Xu, Department of Nutrition, Daping Hospital and Research Institute of Surgery, Third Military Medical University, Chongqing 400042, P.R. China

E-mail: hongxiaxu@tmmu.edu.cn

*Contributed equally

Key words: Platycodin D, breast cancer, MDM2, MDMX, p53 exhibited changes similar to those observed in vitro. These findings indicate that $\mathrm{PD}$ exerted in vitro and in vivo anticancer effects against MDA-MB-231 breast cancer cells, that PD is a potential MDM2/MDMX inhibitor, and that the anticancer effects of PD were likely associated with its inhibition of these proteins. Our observations help to identify a mechanism by which PD functions as an anti-breast cancer agent.

\section{Introduction}

Breast cancer is the most frequently diagnosed malignancy and the leading cause of cancer death among females worldwide, accounting for $25 \%$ of all cancer cases and $15 \%$ of the total cancer deaths in 2012 (1). According to the American Cancer Society, about 231,840 new cases of invasive breast cancer and approximately 60,290 new cases of carcinoma in situ would occur in the US in 2015 (2). To relieve the huge burden of cancer therapy and promote women's health, there is an urgent need to discover new drugs to treat breast cancer.

Natural products, such as thymoquinone, wogonin, naphtho, and quercetin, have been shown to possess different anticancer functions, such as the induction of cell cycle arrest, suppression of tumor angiogenesis, and inhibition of cell migration or invasion (3). Platycodin D (PD), a triterpenoid saponin derived from the roots of Platycodin grandiflorum, has been reported to possess a wide rangeof health benefits, such as anti-atherosclerotic (4), anti-inflammatory $(5,6)$, hypocholesterolemic, and anti-obesity effects (7), as well as spermicidal and contraceptive activity (8). Among the various health benefits of PD, anticancer effects have been noted in several cancer cell lines, including lung cancer cells (9), hepatocellular carcinoma cells (10), gastric cancer cells (11), prostate cancer cells (12), and leukemia cells $(13,14)$. PD has also been reported to inhibit the viability of human breast cancer cells in vitro (15-17).

The mouse double minute-2 ( $m d m 2)$ gene, which was discovered as an oncogene in 1991 (18), has been reported to be amplified in more than 40 different types of malignancies. As reported by Rayburn et al (19), the protein encoded by $m d m 2$, MDM2, is associated with cancer development, progression, the resistance to chemotherapy, and can serve 
as a prognostic marker (20). The tumor suppressor p53 acts as a transcription factor regulating genes involved in DNA repair, cell cycle arrest and apoptosis. The protein function of wild-type p53 gene may be lost because of overexpression of MDM2/MDMX. The inhibition of MDM2-p53 interaction presents an appealing therapeutic strategy for the treatment of cancer (21). As is well known, the p53 gene is mutated in $\sim 50 \%$ malignant tumors (22). There are distinct functional differences between mutant and wild-type p53. Mutant p53 loses its tumor suppressive activity and may actually function as an oncogene (23), which is widely expressed on the MDA-MB-231 human breast cancer cell line (23). The highly metastatic MDA-MB-231 cells are special cells in that they are triple-negative breast cancer cells lacking the expression of the estrogen receptor, progesterone receptor, and human epidermal growth factor receptor 2 . These cells are insensitive to many types of chemotherapy and radiation, mimicking the clinical picture, where there are fewer or no effective treatment options compared to non-triple-negative disease. Since PD has been shown to exert anticancer effects in many types of malignant cells, we wondered whether PD exerts its functions in inhibiting tumor growth by inhibiting MDM2 and mutant p53 in MDA-MB-231 cells.

In the present study, we first evaluated the effects of PD on the viability, proliferation, and cell cycle of MDA-MB-231 human breast cancer cells. MDA-MB-231 xenograft tumors were subsequently established to assess the functions of PD in vivo. We also detected the expression of G0/G1 phaserelated proteins, as well as MDM2, MDMX, mutant p53, p21, and p27 in vitro and in vivo to aid in our understanding of the mechanism by which PD exerts these effects. We believe that the present data support further investigations into the application of PD as a safe and effective natural compound for the treatment of triple-negative breast cancer.

\section{Materials and methods}

Chemicals and reagents. Compound PD (purity: >98\%) was purchased from Chengdu MustBio-Technology Co., Ltd. (Chengdu, China). The structure and molecular weight of PD were shown in Fig. 1A. RPMI-1640, fetal bovine serum (FBS), trypsin, and phosphate-buffered saline (PBS) were obtained from Hyclone (Carlsbad, CA, USA). Dimethyl sulfoxide (DMSO) and 3-(4,5-dimethylthiazol-2-yl)-2,5-diphenyltetrazolium bromide (MTT) were procured from Sigma-Aldrich (St. Louis, MO, USA). The BrdU Cell Proliferation Assay kit was purchased from Cell Signaling Technology Inc. (Danvers, MA, USA). The antibodies against human CDK2, CDK4, CDK6, and Cyclin E used in the western blot analyses were obtained from Boster Biotechnology (Wuhan, China). The anti-Ki67 antibody was from Abcam (Burlingame, CA, USA). The mouse anti-Flag antibody (F-3165) was from Sigma-Aldrich. The anti-human MDM2 antibody was from Calbiochem (Billerica, MA, USA), while the anti-p27 antibody was procured from Cell Signaling Technology, Inc. The MDMX, p21 and p53 antibodies were purchased from Santa Cruz Biotechnology, Inc. (Santa Cruz, CA, USA). The GAPDH antibody was obtained from Good Here Biotechnology Co. Ltd. (Hangzhou, China) and the secondary antibodies were all purchased from ZSGB-BIO (Beijing, China).
The secondary antibody for immunocytochemistry was obtained from Amyjet Scientific Inc. (Wuhan, China). The MDM2 siRNA and negative control siRNA were obtained from Guangzhou RiboBio Co., Ltd. (Guangzhou, China). The liposome transfection reagent, Lipofectamine 2000, was purchased from Invitrogen Corporation (Waltham, MA, USA).

Cell lines and cell culture. The highly metastatic human breast cancer cell line, MDA-MB-231, was obtained from the Type Culture Collection of the Chinese Academy of Sciences (Shanghai, China), and cells were cultured in RPMI-1640 containing $10 \%$ FBS. The cells were maintained in a humidified incubator at $37^{\circ} \mathrm{C}$ containing $5 \% \mathrm{CO}_{2}$ and were passaged at a 1:2 dilution upon confluence every two to three days.

Cell viability assay. The MTT assay was used to assess the impact of PD treatment on the viability of MDA-MB-231 cells. To determine whether PD had a concentrationdependent effect on the cell viability, we designed a concentration gradient from 0 to $20 \mu \mathrm{M}$ of PD. The cells were seeded into 96-well plates at a density of $1 \times 10^{4}$ cells per well. After being cultured in an incubator overnight, the cells were treated with various concentrations of $\mathrm{PD}(0,2.5,5,10$ or $20 \mu \mathrm{M}$ ) for $48 \mathrm{~h}$ then $5 \mathrm{mg} / \mathrm{ml}$ of MTT solution was added into each well. After the cells were incubated with the MTT solution for $4 \mathrm{~h}$, DMSO was used to dissolve the formazan crystals, and the absorbance was read at $570 \mathrm{~nm}$ using a Bio-Tek Synergy HT multifunction plate reader (BioTek, Winooski, VT, USA). Three independent experiments were conducted to determine the $\mathrm{IC}_{50}$ value of PD.

Immunocytochemistry. MDA-MB-231 cells were exposed to 1/1,000 DMSO or $20 \mu \mathrm{M}$ PD for $48 \mathrm{~h}$ to observe the expression of the proliferation marker, Ki67. After being exposed to DMSO or PD, the cells were fixed with $4 \%$ paraformaldehyde for $30 \mathrm{~min}$, incubated sequentially with $0.3 \%$ Triton X-100 for $1 \mathrm{~h}$, goat serum for $30 \mathrm{~min}$, and an anti-Ki67 (dilution ratio $1: 1,000)$ antibody overnight at $4^{\circ} \mathrm{C}$. After that, the sections were rinsed with $\mathrm{PBS}$, incubated with a $\mathrm{Cy} 3$-conjugated AffiniPure Donkey Anti-Rabbit IgG $(\mathrm{H}+\mathrm{L})$ secondary antibody, and stained with DAPI solution. Finally, the cells were observed and photographed under a fluorescence microscope.

Cell proliferation assay. To evaluate the effects of $\mathrm{PD}$ on the proliferation of MDA-MB-231 cells, we performed the BrdU Cell Proliferation Assay. Cells ( $1 \times 10^{4}$ cells per well) were seeded into 96-well plates and treated with PD at concentrations of $0,2.5,5,10$, or $20 \mu \mathrm{M}$ for $48 \mathrm{~h}$. The cells were then exposed to BrdU label for another $10 \mathrm{~h}$, fixed for $30 \mathrm{~min}$, and incubated with an anti-BrdU antibody for $1 \mathrm{~h}$ at room temperature, then treated according to the manufacturer's instructions. Finally, the absorbance was read at dual wavelengths of 450 and $540 \mathrm{~nm}$ by a Bio-Tek Synergy HT multifunction plate reader (BioTek).

Cell cycle analysis. To assess the effects of PD on the cell cycle of MDA-MB-231 cells, we performed a cell cycle analysis using flow cytometry. The cells were seeded into $50 \mathrm{ml}$ culture bottles at a density of $1-2 \times 10^{6}$ cells per bottle and were treated with different concentrations of $\operatorname{PD}(0,2.5,5$, 


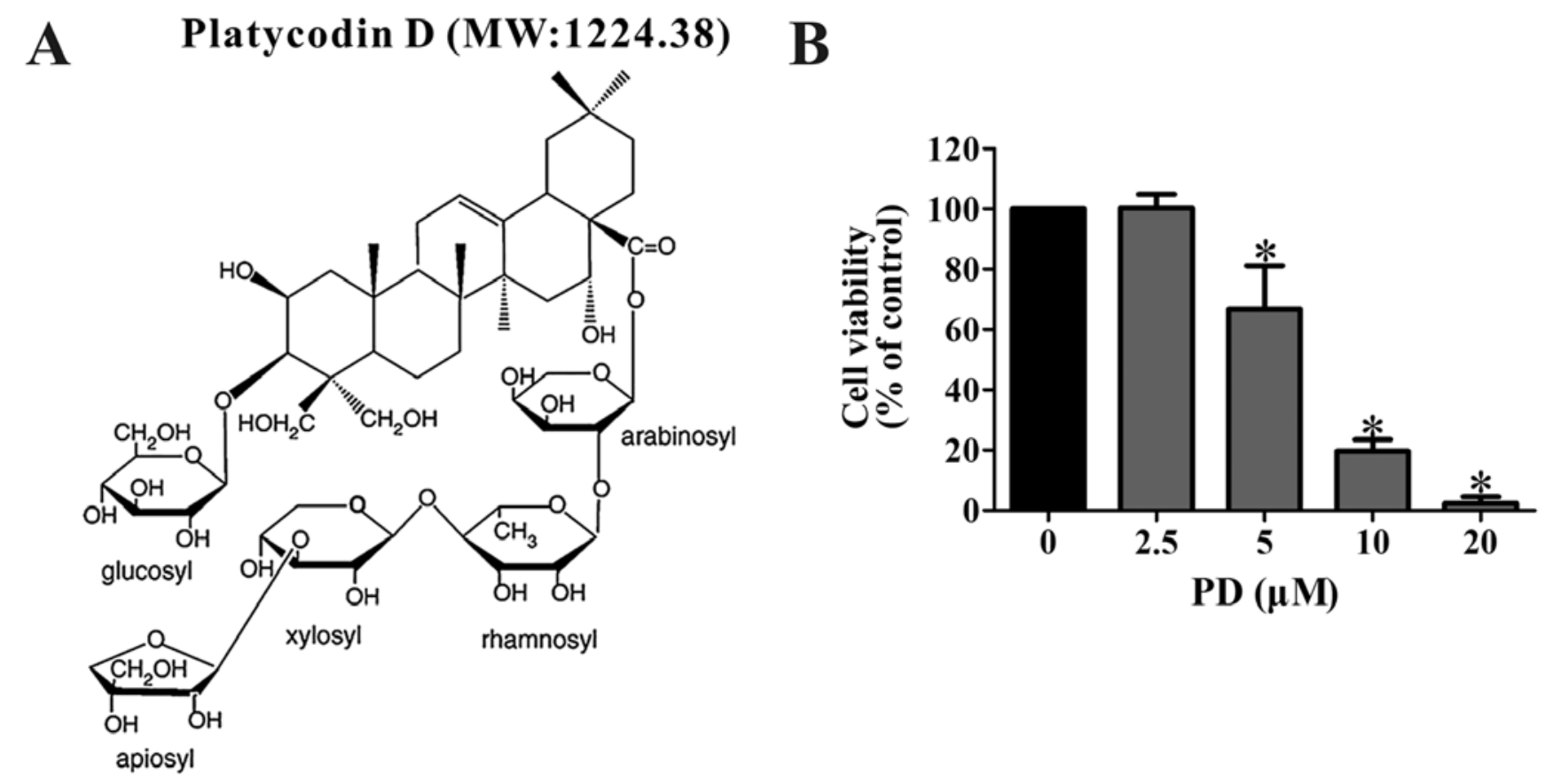

Figure 1. Effects of PD on the viability of MDA-MB-231 cells. (A) The chemical structure and molecular weight of PD. (B) For the MTT assay, cells were treated with $2.5,5,10$ or $20 \mu \mathrm{M}$ of PD for $48 \mathrm{~h}$. Three independent experiments were performed to calculate the $\mathrm{IC}_{50}$. ( $(\mathrm{p}<0.05)$.

$10,20 \mu \mathrm{M})$. After being incubated with the various concentrations of PD for $48 \mathrm{~h}$, we collected the cells by centrifugation, washed them twice with cold PBS, and then fixed them with $75 \%$ ethanol overnight at $4^{\circ} \mathrm{C}$. The next day, the cells were resuspended with $100 \mu \mathrm{g} / \mathrm{ml}$ RNAase and $50 \mu \mathrm{g} / \mathrm{ml}$ PI staining solution. The DNA contents were detected by a FACSCaliber flow cytometer (BD Biosciences, San Jose, CA, USA).

Western blot analysis. MDA-MB-231 cells were exposed to various concentrations of PD $(0,5,10$ and $20 \mu \mathrm{M})$ for $24 \mathrm{~h}$. The cells were then lysed with RIPA buffer containing $1 \%$ PMSF. Equal amounts of protein were subjected to $8-12 \%$ SDS-PAGE. After being electrophoresed for $2 \mathrm{~h}$, the separated proteins were transferred to PVDF membranes which were blocked in $5 \%$ skim milk for $4 \mathrm{~h}$ at $37^{\circ} \mathrm{C}$, and then were incubated with specific antibodies overnight at $4^{\circ} \mathrm{C}$ with gentle shaking. After being washed with TBST solution for $40 \mathrm{~min}$, the membranes were incubated with goat anti-mouse/rabbit horseradish peroxidase-conjugated secondary antibodies for $2 \mathrm{~h}$ at $37^{\circ} \mathrm{C}$, followed by exposure of the membranes to film in a darkroom.

Transfection experiments using an MDM2 plasmid. The pcDNA3-Flag-MDM2 plasmid was provided by Dr Ruiwen Zhang, Texas Tech University Health Sciences Center (Amarillo, TX, USA). MDA-MB-231 cells were transfected with $3 \mu \mathrm{g}$ pcDNA3-Flag-MDM2 plasmid for 5-7 $\mathrm{h}$ and were then treated with PD $(20 \mu \mathrm{M})$ for $24 \mathrm{~h}$. The procedure was carried out as previously described (12). Western blot analysis was performed to assess the expression of various proteins.

Transient transfection of MDM2 siRNA. MDA-MB-231 cells were transfected with MDM2 siRNA at a final concentration of $100 \mathrm{nM}$ or with $50 \mathrm{nM}$ of a negative control siRNA for 5-7 h.
Then, $20 \mu \mathrm{M}$ of PD was added to the MDM2-silenced cells for 24 h. Finally, a western blot analysis was performed to detect the expression of MDM2-related proteins. To verify whether PD inhibited highly metastatic MDA-MB-231 breast cancer growth by targeting the MDM2 oncogene, we performed the MTT and BrdU assays on cells transfected with MDM2 siRNA or an MDM2 expression plasmid.

Tumor xenograft study. Female athymic BALB/C nude (nu/nu) mice (4-6 weeks old) were procured from the Medical Experimental Animal Center of the Third Military Medical University. The animal care and experimental procedures were performed with the approval of the Animal Ethics Committee of the Third Military Medical University. MDA-MB-231 cells were collected and resuspended in serum-free RPMI-1640 medium and were mixed with Matrigel (BD Biosciences, Bedford, MA, USA) at a 3:1 ratio. Each mouse was subcutaneously injected with $5 \times 10^{6}$ cells per mouse in the right subaxillary area to establish a MDA-MB-231 breast cancer xenograft model. Six days after the injection of the cells, the animals bearing human breast cancer xenograft tumors were randomly divided into a control group, $1 \mathrm{mg} / \mathrm{kg}$ PD group and $2.5 \mathrm{mg} / \mathrm{kg}$ PD group. PD was dissolved in PEG400:Saline:Ethanol (400:300:200, $\mathrm{v} / \mathrm{v} / \mathrm{v}$ ) and was administered via i.p. injection. All mice were treated five days per week for four weeks. The body weight and tumor size of each mouse was monitored every three days by an electronic scale and Vernier calipers by two different observers. We measured two perpendicular diameters of the xenograft tumors: the longer dimension was recorded as ' $a$ ' and the shorter dimension was recorded as ' $b$ '. We converted the two measured values into the tumor weight using the formula: $\left(\mathrm{axb}^{2}\right) / 2$. The mice were sacrificed after being treated for four weeks. The xenograft tumors were carefully removed, 
A
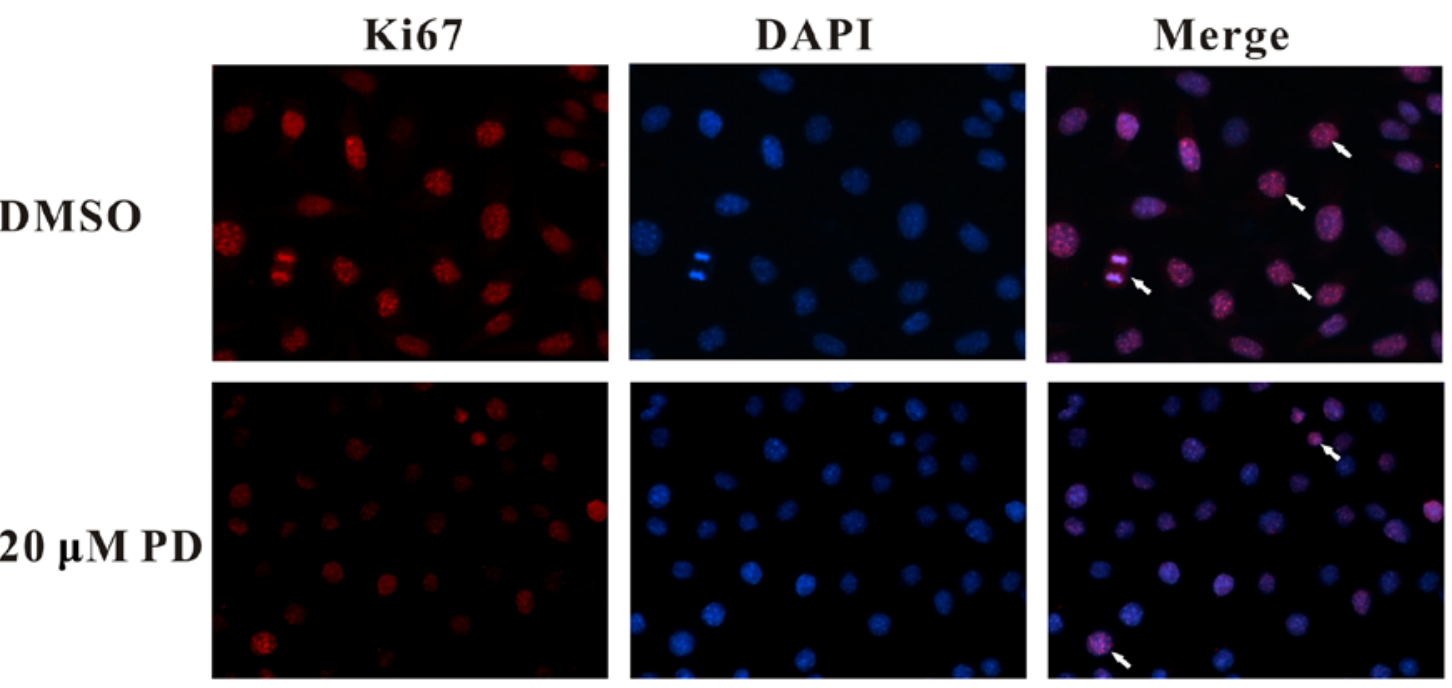

B
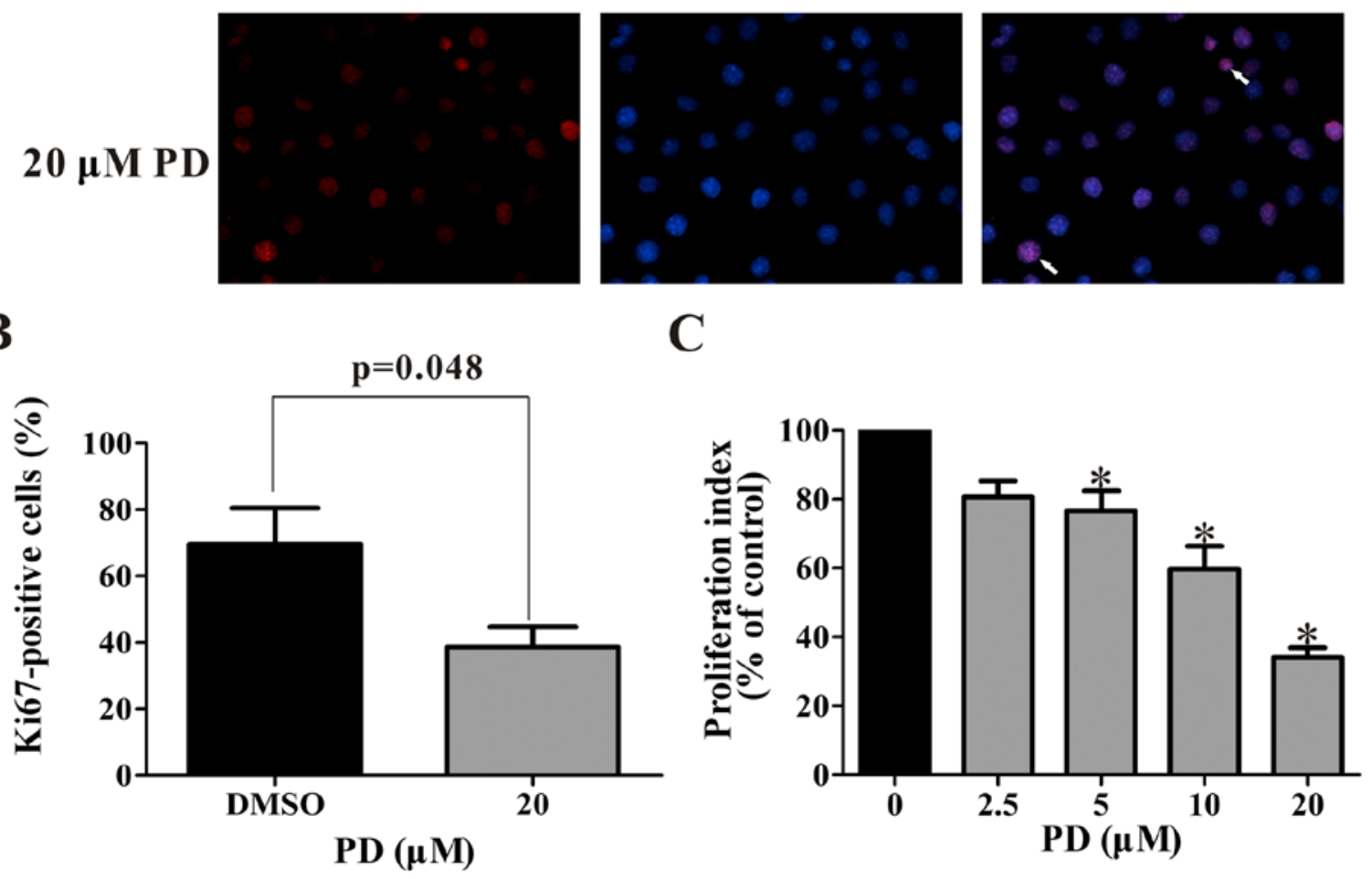

Figure 2. PD inhibited the proliferation of MDA-MB-231 cells. (A) The expression of Ki67 in cells was detected by immunocytochemistry. Cells were treated with DMSO or $20 \mu \mathrm{M}$ of PD for $48 \mathrm{~h}$, then were incubated with an anti-Ki67 antibody and corresponding secondary antibody. The Ki67-positive cells are indicated by solid arrows. (B) Ki67-positive cells were counted three times in at least four high power fields by two evaluaters. Three separate experiments were performed to detect the expression of Ki67. PD decreased the proportion of proliferating cells from 69.5 to $38.6 \%$. (C) Cells were exposed to 2.5, 5, 10, or $20 \mu \mathrm{M}$ of PD for $48 \mathrm{~h}$ for the BrdU cell proliferation assay. PD significantly inhibited the cell proliferation at concentrations $>5 \mu \mathrm{M}$.

weighed, photographed, and frozen at $-80^{\circ} \mathrm{C}$ until used for western blot analysis.

Statistical analysis. Graphic illustrations of the results were obtained using the Graphpad Prism 5.0 software program (GraphPad Software, San Diego, CA, USA). SPSS 17.0 was used to perform the statistical analysis. Measured data were expressed as the means \pm standard deviations (SD) and the statistical significance of differences between groups was assessed using a one-way ANOVA when the data were normally distributed and had homogeneous variance. A value of $p<0.05$ was considered to be statistically significant.

\section{Results}

$P D$ inhibits the growth of MDA-MB-231 breast cancer cells in vitro. We performed three independent MTT assays to assess the viability of MDA-MB-231 cells following PD treatment. The cells were treated with various concentrations of PD $(0,2.5,5,10$, and $20 \mu \mathrm{M})$ for $48 \mathrm{~h}$ then the survival percentages of the cells were measured. As shown in Fig. 1B, these results indicated that the $\mathrm{IC}_{50}$ value for $\mathrm{PD}$ was $7.77 \pm 1.86 \mu \mathrm{M}$ and $\mathrm{PD}$ significantly decreased the viability of the MDA-MB-231 cells in a concentration-dependent manner.

$P D$ decreases the proliferation of the MDA-MB-231 breast cancer cells in vitro. Cell proliferation was detected by immunocytochemistry and BrdU cell proliferation assay. The expression of the proliferation marker Ki67, is shown in Fig. 2A and B with immunocytochemistry method. The proportion of proliferating cells was reduced from $69.5 \%$ to $38.6 \%$ by treatment with $20 \mu \mathrm{M}$ PD (Fig. 2B). The BrdU cell proliferation assay indicated that $\mathrm{PD}$ inhibits the proliferation of MDA-MB-231 cells in a concentration-dependent manner (Fig. 2C). All concentrations of PD over $5 \mu \mathrm{M}$ significantly decreased the cell proliferation. In the cells treated with the $20 \mu \mathrm{M}$ concentration of $\mathrm{PD}$, the proliferation index decreased to $34 \pm 0.05 \%$ of that in the control group.

$P D$ induces cell cycle arrest in the G0/G1 phase in MDA-MB231 cells. After treatment with various concentrations of PD for $48 \mathrm{~h}$, the DNA contents of the cells were detected by flow 
A

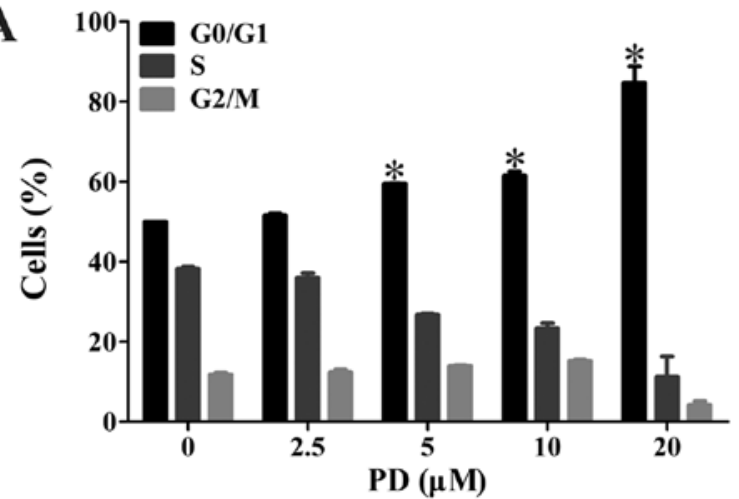

C
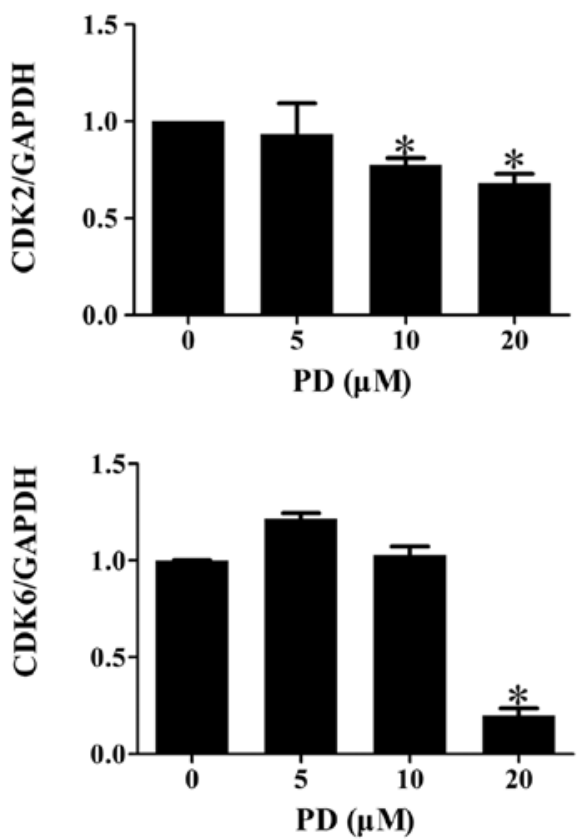

B $\mathrm{PD}(\mu \mathrm{M})$

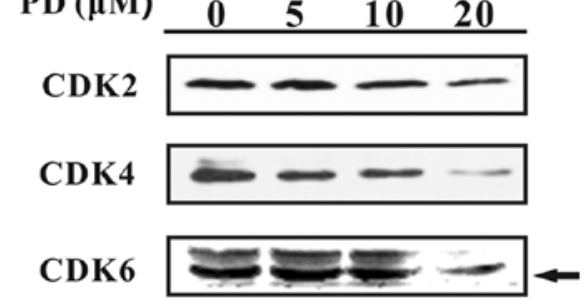

Cyclin E
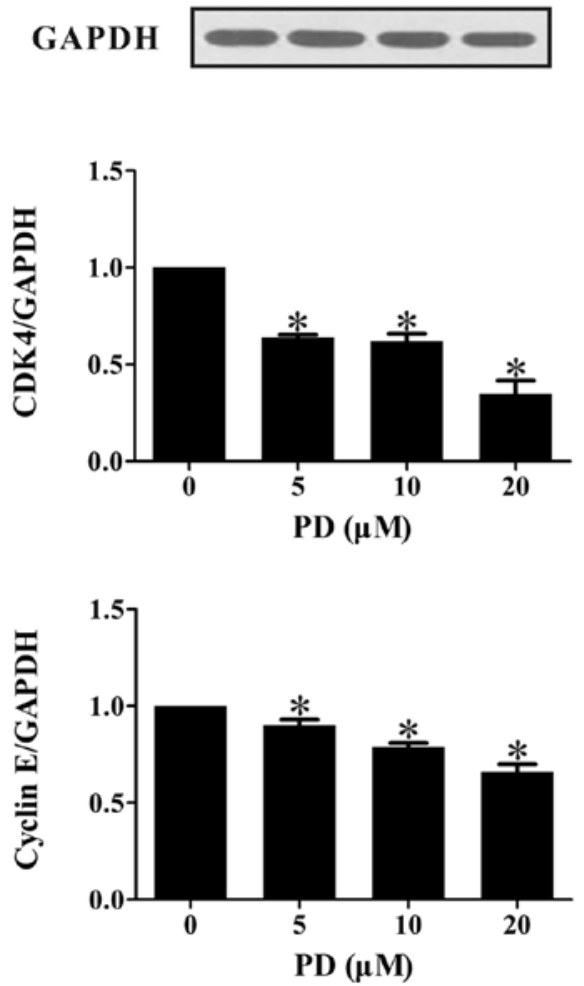

Figure 3. PD induced cell cycle arrest in the G0/G1 phase in MDA-MB-231 cells. (A) Cells were exposed to 2.5, 5, 10 or $20 \mu \mathrm{M}$ of PD for $48 \mathrm{~h}$ and the cell cycle progression was assessed by flow cytometry. The 5, 10 and $20 \mu \mathrm{M}$ concentrations of PD induced significant cell cycle arrest in the G0/G1 phase. (B and C) The expression levels of G0/G1 phase-related proteins was analyzed by western blotting after treatment with various concentrations of PD for 24 h. PD downregulated the expression of CDK2, CDK4, CDK6, and Cyclin E. ("p<0.05).

cytometry. As shown in Fig. 3A, the majority of the MDA-MB231 cells were blocked at the G0/G1 phase following PD treatment, and this occurred in a concentration-dependent manner. Treatment with $20 \mu \mathrm{M}$ PD significantly arrested the cell cycle progression, with $84.67 \pm 4.13 \%$ of cells found in the $\mathrm{G} 0 / \mathrm{G} 1$ phases after a $48 \mathrm{~h}$ treatment.

We also performed western blot assays to detect the expression of G0/G1 phase-related proteins. As illustrated in Fig. 3B and C, PD significantly decreased the expression levels of CDK2, CDK4, CDK6, and Cyclin E.

$P D$ changes the expression levels of MDM2, MDMX, mutant $p 53, p 21$ and $p 27$. After the cells were treated with PD for $24 \mathrm{~h}$, the expression levels of MDM2, MDMX, mutant p53, p21 and p27 were analyzed by western blotting. As shown in Fig. 4A, PD downregulated the expression of MDM2, MDMX and mutant p53. In contrast, PD upregulated the expression levels of p21 and p27 (Fig. 4B), which are proteins downstream of MDM2 and MDMX, which function as cancer suppressors.
To further understand the expression of MDM2-related proteins, we performed a transfection experiment. A pcDNA3Flag-MDM2 plasmid or MDM2 siRNA was used to control the MDM2 expression. As shown in Fig. 5A, the expression of MDM2 was downregulated after treatment with $20 \mu \mathrm{M}$ of PD for $24 \mathrm{~h}$. The upregulation of MDMX and mutant p53 were also inhibited by treatment with PD. The changes in $\mathrm{p} 21$ and p27 expression were the opposite of those of MDM2 (Fig. 5B).

As illustrated in Fig. 6, MDM2 was silenced by MDM2 siRNA and further downregulated by PD. The expression levels of MDMX and mutant p53 were decreased along with the MDM2 expression level, but the p21 and p27 proteins were upregulated.

$P D$ inhibits $M D A-M B-231$ cell growth by targeting the MDM 2 oncogene. As shown in Fig. 7A and $\mathrm{B}$, the cells transfected with the pcDNA3-Flag-MDM2 plasmid showed a similar sensitivity to PD as the parental cells. In contrast, the cells transfected with siRNA against MDM2 showed 
A

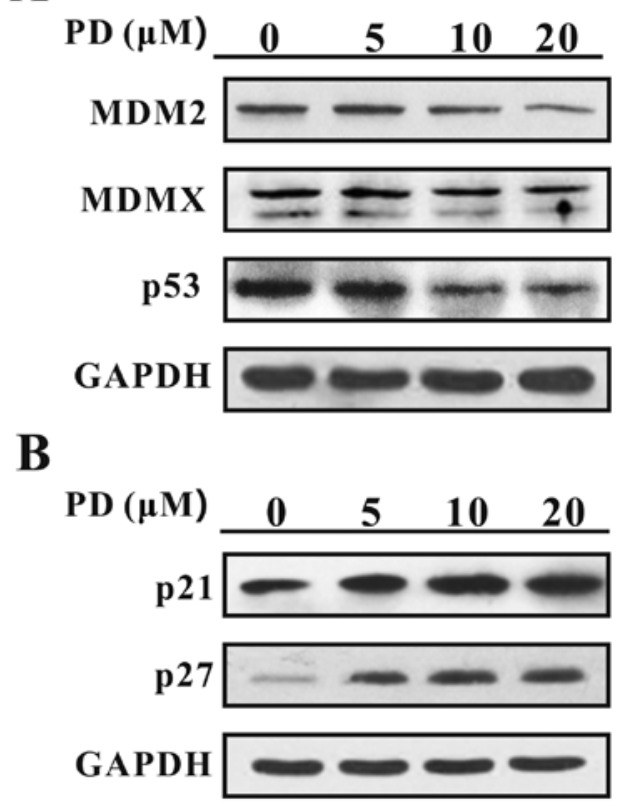

Figure 4. PD decreased the expression of MDM2, MDMX, and mutant p53, and increased the levels of p21 and p27 in MDA-MB-231 cells. Cells were treated with 5,10 or $20 \mu \mathrm{M}$ of PD for $24 \mathrm{~h}$ to detect the expression levels of MDM2 and MDM2-related proteins. (A) PD decreased the expression of MDM2, MDMX and mutant p53. (B) The expression levels of proteins downstream of MDM2/MDMX (p21 and p27) were upregulated by PD, which led to anticancer effects.

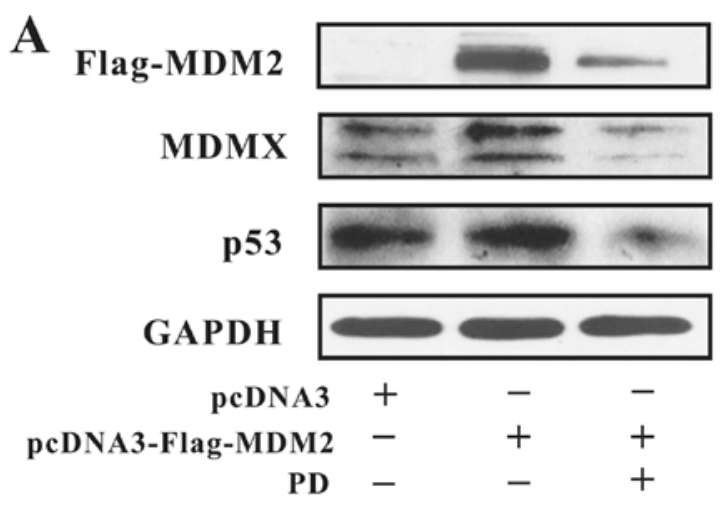

B

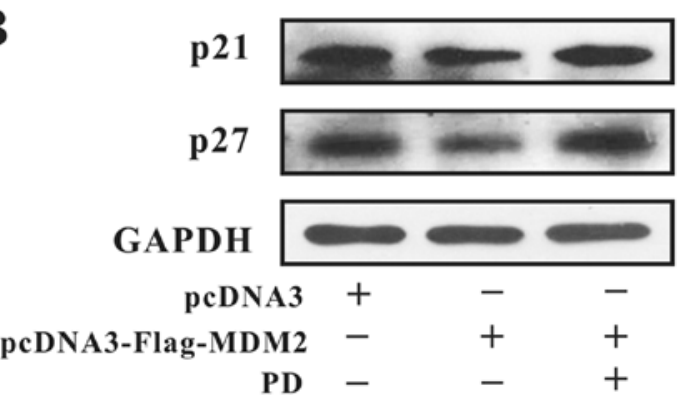

Figure 5. The changes in the expression of proteins in the presence of MDM2 overexpression. A total of $3 \mu \mathrm{g}$ of the pcDNA-Flag-MDM2 or control (pcDNA3) plasmid was transfected into MDA-MB-231 cells to change the expression of the MDM2 protein. Cells were treated with or without $20 \mu \mathrm{M}$ of PD for $24 \mathrm{~h}$, then the expression levels of MDM2-related proteins were detected by western blotting. (A) MDMX and p53 were upregulated in the cells with MDM2 overexpression. However, the upregulated expression of MDMX and mutant p53 were obviously diminished by PD treatment. (B) MDM2 overexpression resulted in the downregulation of p21 and p27, but the expression of these proteins was largely rescued by PD treatment.
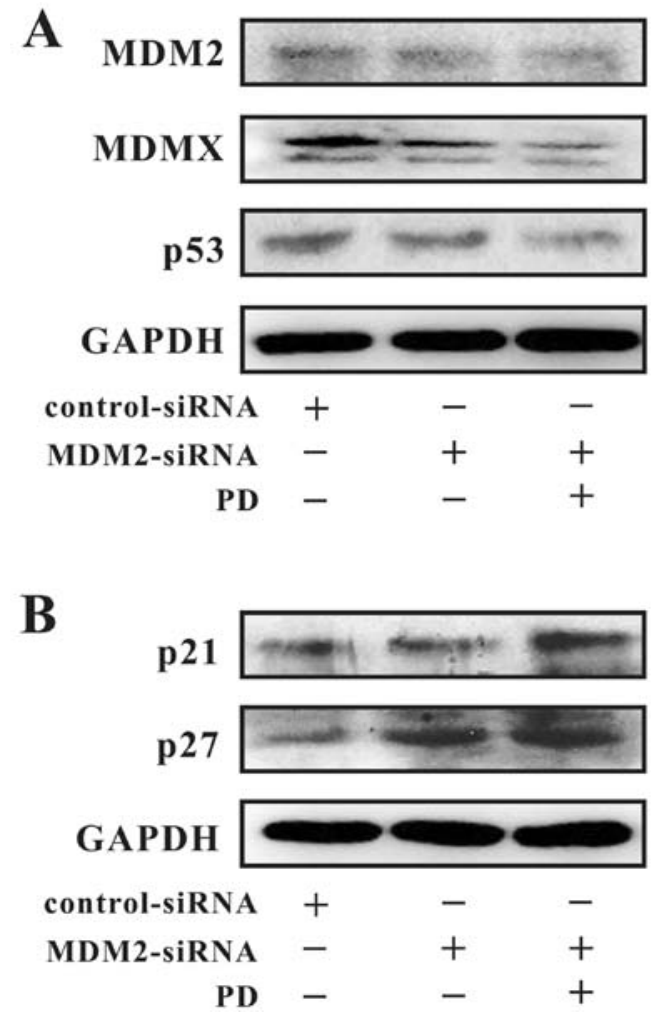

Figure 6. Changes in the expression of proteins when MDM2 is silenced. Cells were transfected with MDM2 siRNA or a control siRNA to inhibit the expression of MDM2. Then, $20 \mu \mathrm{M}$ of PD was added to these cells for $24 \mathrm{~h}$ to observe the changes in the expression of MDM2-related proteins. (A) MDM2 was downregulated by MDM2 siRNA and its expression was further decreased by PD. The expression levels of MDMX and p53 were also decreased by the MDM2 siRNA and were further decreased by PD treatment. (B) The expression levels of p21 and p27 were increased when MDM2 was silenced and were further upregulated by PD treatment.

greater inhibition of the cell viability following treatment with PD than did the parental cells. Similarly, as shown in Fig. 7C and D, the cells transfected with the pcDNA3-Flag-MDM2 plasmid were less sensitive to treatment with PD than were the parental cells, while the cells transfected with siRNA-MDM2 showed a greater decrease in proliferation following treatment with PD than did the parental cells.

Together, these findings indicate that PD affected the MDM2, and the effects are related to its anti-proliferative and anti-growth effects on the cells.

PD inhibits the growth of MDA-MB-231 xenograft tumors. All xenograft tumor-bearing mice were randomly divided into a control group, $1 \mathrm{mg} / \mathrm{kg}$ PD group and $2.5 \mathrm{mg} / \mathrm{kg}$ PD group so that all three groups had a similar xenograft tumor size. Each mouse was treated five days a week for four weeks. The body weight and tumor size were analyzed every three days to estimate the effects of different doses of PD (Fig. 8A and B). As shown in Fig. 8C and D, there were significant differences in the tumor weight among the three treatment groups. PD ( $1 \mathrm{mg} / \mathrm{kg}$ and $2.5 \mathrm{mg} / \mathrm{kg}$ ) inhibited the tumor growth by $24 \%$ and $30 \%$ compared to the control on day $27(\mathrm{p}<0.05)$. However, there were no significant differences in the body weight change among the three treatment groups (Fig. 8A). These results indicated that $\mathrm{PD}$ administration significantly inhibited 
A

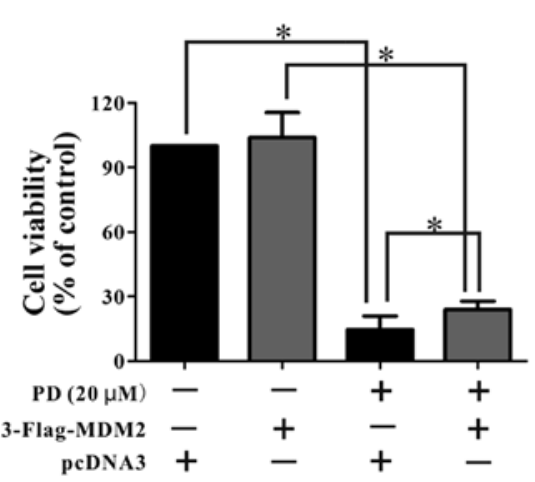

C

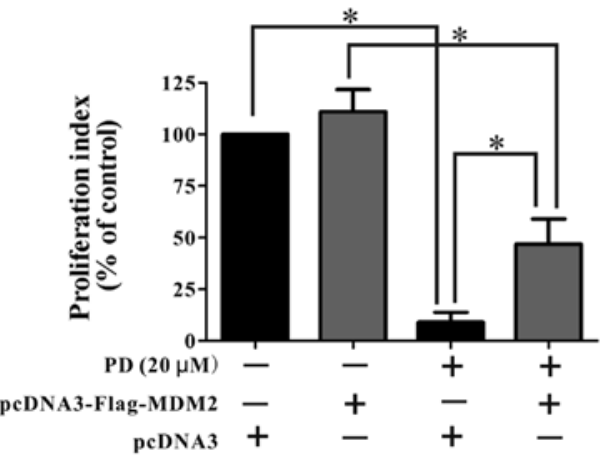

B

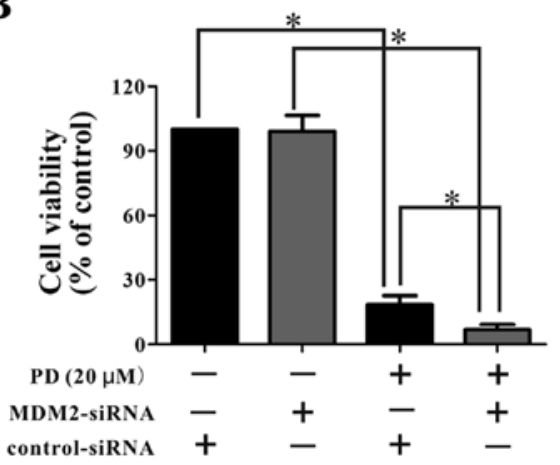

D

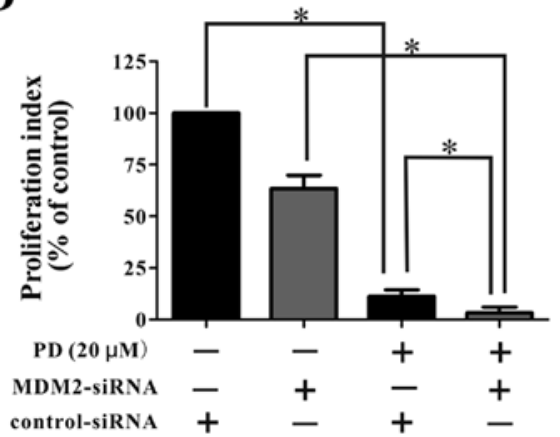

Figure 7. PD inhibited cell viability and proliferation by targeting the MDM 2 oncogene in MDA-MB-231 cells. A $20 \mu \mathrm{M}$ concentration of PD were used to treat pcDNA3-Flag-MDM2 plasmid- or MDM2 siRNA-transfected MDA-MB-231 cells and parental cells (control) to compare the inhibitory effects of PD to determine the dependence on MDM2. (A) The effects of PD on the cell viability were more obvious in the MDM2 siRNA-transfected cells than MDM2plasmid transfected cells and parental cells. (B) The proliferation index of MDM2 siRNA cells was lower than that of the MDM2-overexpressing cells.

A

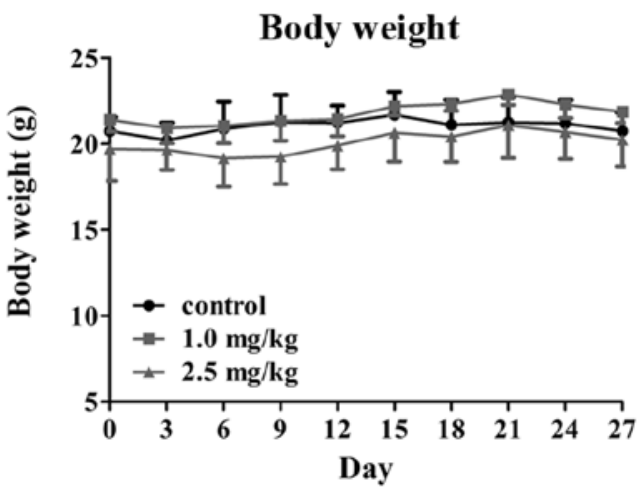

C

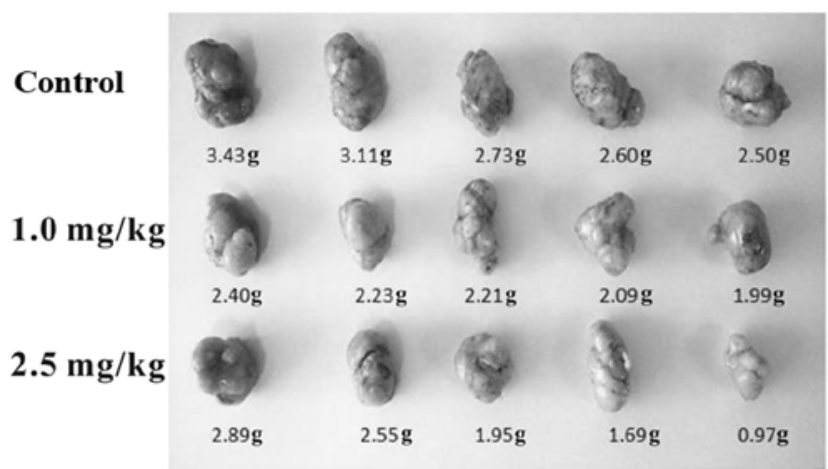

B

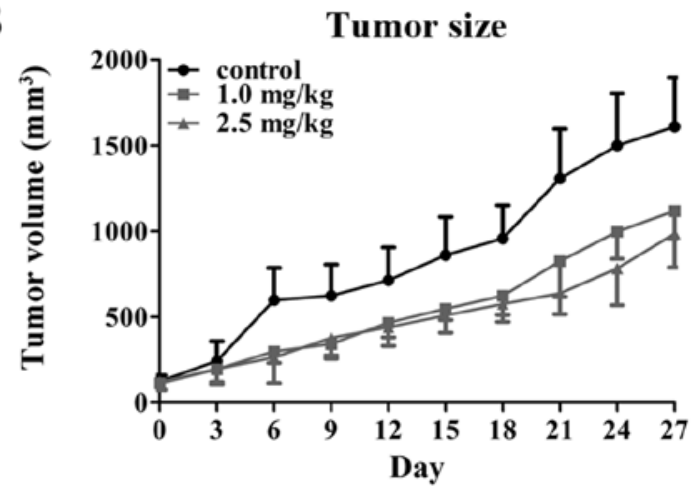

D

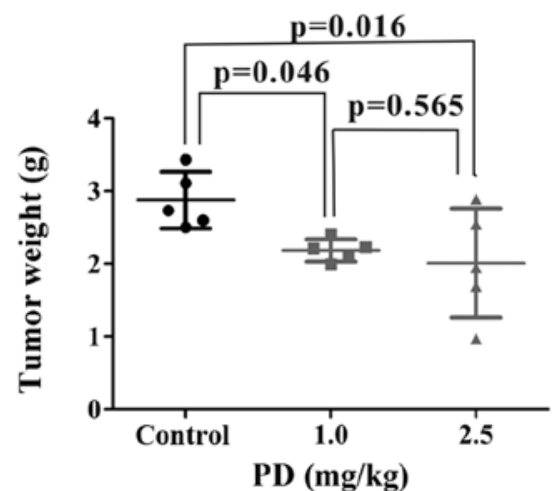

Figure 8. PD inhibited the tumor growth in the MDA-MB-231 xenograft model. Mice were treated with PD at doses of $0,1 \mathrm{or} 2.5 \mathrm{mg} / \mathrm{kg} / \mathrm{day}$ with intraperitoneal injection for four weeks. (A) The body weights of the mice were monitored every three days to assess the potential toxicity of PD. (B) The volumes of the tumors were measured using Vernier calipers every three days to monitor the changes in the xenograft tumors. (C and D) After four weeks of treatment, the xenograft tumors were removed, photographed and weighed. Treatment with $1 \mathrm{mg} / \mathrm{kg}$ and $2.5 \mathrm{mg} / \mathrm{kg}$ of PD significantly inhibited the growth of the xenograft tumors. $(\mathrm{p} p 0.05)$. 

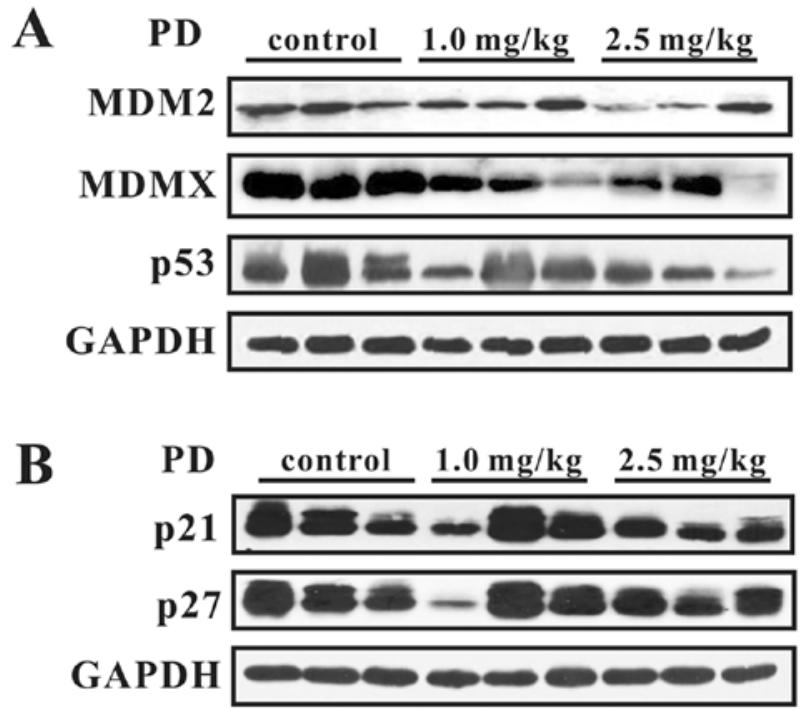

$\mathbf{C}$

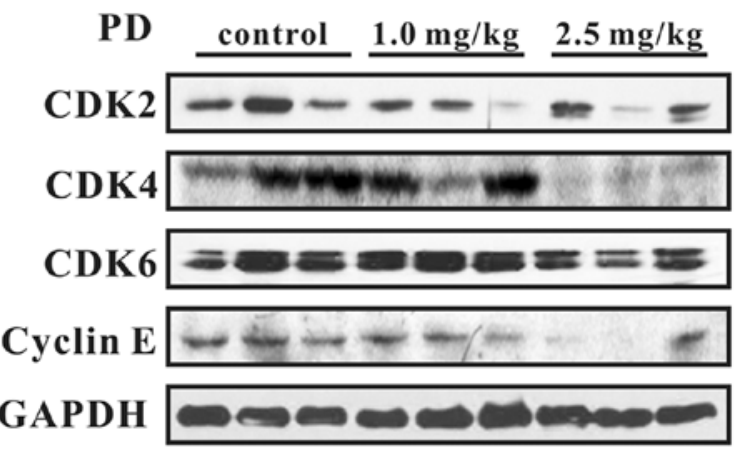

Figure 9. The effects of PD on the expression levels of various proteins in MDA-MB-231 xenograft tumors. Western blotting was performed to assess the expression levels of proteins in the MDM2-MDMX-p53 pathway, downstream proteins and $\mathrm{G} 0 / \mathrm{G} 1$ phase-related proteins in mice after treatment with the three concentrations of PD for four weeks. (A) PD downregulated the expression of the MDM2, MDMX, and mutant p53 proteins. (B) PD upregulated the expression of $\mathrm{p} 21$ and $\mathrm{p} 27$. (C) PD decreased the expression of G0/G1 phase-related proteins (CDK2, CDK4, CDK6 and Cyclin E).

the growth of xenograft tumors even at a dose of $1 \mathrm{mg} / \mathrm{kg}$ body weight. PD treatment did not lead to any significant change in the body weights of the mice up to $2.5 \mathrm{mg} / \mathrm{kg}$, suggesting that PD does not exert any major toxicity at this dose.

We further examined the expression levels of various proteins in vivo. As shown in Fig. 9, PD decreased the expression of MDM2, MDMX, and mutant p53 and increased the expression of p21 and p27. The expression of G0/G1 phase-related proteins, including CDK2, CDK4, CDK6, and Cyclin E, was downregulated. These results were consistent with the in vitro findings.

\section{Discussion}

Natural products have been receiving increasing attention because of their diversity of anticancer effects and relative safety. As the most abundant triterpenoid saponin derived from the roots of Platycodin grandiflorum, Platycodin D (PD), has been reported to show a wide range of health benefits, such as anti-atherosclerotic (4), anti-inflammatory $(5,6)$, hypocholesterolemic and anti-obesity effects (7). Previous studies have shown that PD has obvious anticancer activity against different human malignant cancer cells both in vitro $(10,11,13,14,16,17)$ and in vivo $(9,12,15)$.

Breast cancer is a major public health issue for women worldwide. A previous study reported that PD inhibited the growth, migration and invasion of MDA-MB-231 cells by suppressing the EGFR-mediated Akt and MAPK pathways (15). Furthermore, PD blocked breast cancer-induced bone loss by suppressing osteoclastogenesis (24). It was also found to enhance the anti-proliferative effects of doxorubicin in MCF-7 and MDA-MB-231 breast cancer cells (25). The combination of $\mathrm{PD}$ and osthole inhibited the proliferation and invasion of MDA-MB-231 and 4T1 human breast cancer cells (17). We performed the present studies to better understand the potential mechanisms by which PD decreases the viability of MDA-MB-231 cells.

Our present findings showed that PD decreased the viability of MDA-MB-231 cells and inhibited their proliferation in a concentration-dependent manner. $\mathrm{The}^{\mathrm{IC}_{50}}$ value of PD for inhibiting cell growth was less than $10 \mu \mathrm{M}$, and treatment of cells with $20 \mu \mathrm{M}$ PD decreased the proliferation index to $34 \pm 0.05 \%$ of the rate observed in the control group. These anti-proliferative effects were consistent with those reported in previous studies $(10,12,15,26)$. Furthermore, we detected the expression of Ki67 in MDA-MB-231 cells, which is a classic marker of cell proliferation. We observed that Ki67 expression was significantly reduced by PD, which was also consistent with the previous finding that PD could inhibit the expression of Ki67 in MDA-MB-231 xenograft tumors (15).

The results of the cell cycle analysis indicated that PD induced concentration-dependent cell cycle arrest in the G0/G1 phase. It is interesting that PD may have different effects on the cell cycle in different cell lines. As we reported previously, PD induced cell cycle arrest in the G0/G1 phase in the DU145 and $\mathrm{LnCaP}$ prostate cancer cell lines, but induced arrest in the $\mathrm{G} 2 / \mathrm{M}$ phase in the PC3 prostate cancer cell line (12).

We further explored the antitumor effects and potential mechanism of action of PD in MDA-MB-231 cells in vitro and in vivo. MDA-MB-231 cells are triple-negative (ER/PR/ HER2-neg), providing a model of a more difficult-to-treat type of breast cancer. A previous in vitro study on PD in MDA-MB-231 cells reported that PD inhibited the growth of cells via suppression of the EGFR-mediated Akt and MAPK pathways (15). In our study, we focused on the effects of $\mathrm{PD}$ on the expression of mutant p53 and MDM2/MDMX in MDA-MB-231 cells. The tumor suppressor p53, a classic transcription factor, plays an important role in regulating many key cellular processes. When DNA is damaged, p53 regulates a large number of target genes related to cell cycle arrest, apoptosis, senescence, autophagy and metabolism $(27,28)$. However, p53 is mutated in the majority of human cancers, resulting in a prevention or reversal of its functions (29-31). It has been reported that mutated p53 contributes to various steps of tumorigenesis (32). It has been reported that $60-88 \%$ of advanced breast cancers harbor mutant p53. In a previous study, Kim et al found that the levels of mutant p53 could be decreased in a concentration- and time-dependent manner after treatment with a ginsenoside, Rg3, in MDA-MB-231 cells (33). Our present data showed that PD inhibited MDA-MB-231 cell viability by targeting mutant $\mathrm{p} 53$, which suggests that $\mathrm{PD}$ 
represents a safe and effective natural compound that can be used for the treatment of advanced breast cancer.

MDM2 is a well-known oncogene. The p53-MDM2-MDMX loop has been reported as a target for cancer therapy (34). MDM2 and MDMX are important negative regulators of p53 that have been shown to function collaboratively (35). In cancer patients with tumors harboring mutant p53 or without p53 expression, including breast cancer patients, MDM2 overexpression is still found to be involved in cancer growth and metastasis (36). The MDA-MB-231 cells have mutant p53 $(37,38)$, which works as a driving oncogene (39). Therefore, we detected the expression levels of the MDM2, MDMX and mutant p53 proteins in the present study. The results of the western blot analyses indicated that PD can downregulate MDM2, MDMX and mutant p53 and upregulate the expression of p21 and p27. The results of the MTT and BrdU assays in the MDM2 siRNA- and MDM2 plasmid-transfected cells indicated that PD inhibited MDA-MB-231 cell growth by targeting the MDM2 oncogene. Based on these findings, we believe that the effects of PD are at least partially p53-independent.

We further explored the antitumor activity of PD using the MDA-MB-231 xenograft tumor model. We found that PD decreased the growth of the xenograft tumors even at the $1 \mathrm{mg} / \mathrm{kg}$ dose, which was much lower than the dose used in a previous study of the effects of PD on MDA-MB-231 xenograft tumors (15). We also checked the protein expression of mutant p53, MDM2, MDMX, p21 and p27 in the xenograft tumor tissues, and found that all of the differences in expression between control and treated animals corresponded with those noted in the in vitro study.

In conclusion, our present study results indicate that $\mathrm{PD}$ can inhibit the growth of MDA-MB-231 human breast cancer cells and tumors, and these effects may be at least partially mediated via its targeting of the MDM2 oncogene. PD decreased the expression levels of MDM2, MDMX, and mutant p53, and upregulated the expression of proteins downstream of MDM2 (p21 and p27) to induce cell cycle arrest, inhibit cell proliferation, and promote cell death.

It is a limitation to draw our conclusion based on the effects on only one cell line (MDA-MB-231 cells). Thefore, detailed mechanisms by which PD inhibits the growth of human triple-negative breast cancer cells will need to be elucidated or confirmed in future studies. However, our findings and those of previous studies suggest that PD may represent a potential anticancer agent for use against advanced breast cancer.

\section{Acknowledgements}

This work was supported by an NSFC grant from the National Natural Science Foundation of China (no. 81171991) to Hong-Xia Xu.

\section{References}

1. Torre LA, Bray F, Siegel RL, Ferlay J, Lortet-Tieulent J and Jemal A: Global cancer statistics, 2012. CA Cancer J Clin 65: 87-108, 2015

2. Ward EM, DeSantis CE, Lin CC, Kramer JL, Jemal A, Kohler B, Brawley OW and Gansler T: Cancer statistics: Breast cancer in situ. CA Cancer J Clin 65: 481-495, 2015.
3. Safdari Y, Khalili M, Ebrahimzadeh MA, Yazdani Y and Farajnia S: Natural inhibitors of PI3K/AKT signaling in breast cancer: Emphasis on newly-discovered molecular mechanisms of action. Pharmacol Res 93: 1-10, 2015

4. Wu J, Yang G, Zhu W, Wen W, Zhang F, Yuan J and An L: Antiatherosclerotic activity of platycodin D derived from roots of Platycodon grandiflorum in human endothelial cells. Biol Pharm Bull 35: 1216-1221, 2012.

5. Chung JW, Noh EJ, Zhao HL, Sim JS, Ha YW, Shin EM, Lee EB, Cheong CS and Kim YS: Anti-inflammatory activity of prosapogenin methyl ester of platycodin $\mathrm{D}$ via nuclear factor-kappaB pathway inhibition. Biol Pharm Bull 31: 2114-2120, 2008.

6. Zhang T, Yang S, Du J, Jinfu Y and Shumin W: Platycodin D attenuates airway inflammation in a mouse model of allergic asthma by regulation NF- $\mathrm{NB}$ pathway. Inflammation 38: 1221-1228, 2015

7. Zhao HL, Harding SV, Marinangeli CP, Kim YS and Jones PJ: Hypocholesterolemic and anti-obesity effects of saponins from Platycodon grandiflorum in hamsters fed atherogenic diets. J Food Sci 73: H195-H200, 2008.

8. Lu Z, Wang L, Zhou R, Qiu Y, Yang L, Zhang C, Cai M, Mi M and $\mathrm{Xu} \mathrm{H}$ : Evaluation of the spermicidal and contraceptive activity of Platycodin D, a Saponin from Platycodon grandiflorum. PLoS One 8: e82068, 2013.

9. Park JC, Lee YJ, Choi HY, Shin YK, Kim JD and Ku SK: In vivo and in vitro antitumor effects of platycodin $\mathrm{d}$, a saponin purified from platycodi radix on the h520 lung cancer cell. Evid Based Complement Alternat Med 2014: 478653, 2014.

10. Li T, Xu WS, Wu GS, Chen XP, Wang YT and Lu JJ: Platycodin D induces apoptosis, and inhibits adhesion, migration and invasion in HepG2 hepatocellular carcinoma cells. Asian Pac J Cancer Prev 15: 1745-1749, 2014.

11. Chun J, Joo EJ, Kang M and Kim YS: Platycodin D induces anoikis and caspase-mediated apoptosis via p38 MAPK in AGS human gastric cancer cells. J Cell Biochem 114: 456-470, 2013.

12. Zhou R, Lu Z, Liu K, Guo J, Liu J, Zhou Y, Yang J, Mi M and $\mathrm{Xu} \mathrm{H}$ : Platycodin $\mathrm{D}$ induces tumor growth arrest by activating FOXO3a expression in prostate cancer in vitro and in vivo. Curr Cancer Drug Targets 14: 860-871, 2015.

13. Kim MO, Moon DO, Choi YH, Shin DY, Kang HS, Choi BT, Lee JD, Li W and Kim GY: Platycodin D induces apoptosis and decreases telomerase activity in human leukemia cells. Cancer Lett 261: 98-107, 2008.

14. Kim MO, Moon DO, Choi YH, Lee JD, Kim ND and Kim GY: Platycodin D induces mitotic arrest in vitro, leading to endoreduplication, inhibition of proliferation and apoptosis in leukemia cells. Int J Cancer 122: 2674-2681, 2008.

15. Chun $\mathrm{J}$ and Kim YS: Platycodin D inhibits migration, invasion, and growth of MDA-MB-231 human breast cancer cells via suppression of EGFR-mediated Akt and MAPK pathways. Chem Biol Interact 205: 212-221, 2013.

16. Yu JS and Kim AK: Platycodin D induces apoptosis in MCF-7 human breast cancer cells. J Med Food 13: 298-305, 2010.

17. Ye Y, Han X, Guo B, Sun Z and Liu S: Combination treatment with platycodin $\mathrm{D}$ and osthole inhibits cell proliferation and invasion in mammary carcinoma cell lines. Environ Toxicol Pharmacol 36: 115-124, 2013.

18. Fakharzadeh SS, Trusko SP and George DL: Tumorigenic potential associated with enhanced expression of a gene that is amplified in a mouse tumor cell line. EMBO J 10: 1565-1569, 1991.

19. Rayburn ER, Ezell SJ and Zhang R: Recent advances in validating MDM2 as a cancer target. Anticancer Agents Med Chem 9: 882-903, 2009.

20. Onel $\mathrm{K}$ and Cordon-Cardo C: MDM2 and prognosis. Mol Cancer Res 2: 1-8, 2004.

21. Nag S, Qin J, Srivenugopal KS, Wang M and Zhang R: The MDM2-p53 pathway revisited. J Biomed Res 27: 254-271, 2013.

22. Brosh R and Rotter V: When mutants gain new powers: News from the mutant p53 field. Nat Rev Cancer 9: 701-713, 2009.

23. Epstein CB, Attiyeh EF, Hobson DA, Silver AL, Broach JR and Levine AJ: p53 mutations isolated in yeast based on loss of transcription factor activity: Similarities and differences from p53 mutations detected in human tumors. Oncogene 16: 2115-2122, 1998.

24. Lee SK, Park KK, Kim HJ, Kim KR, Kang EJ, Kim YL, Yoon H, Kim YS and Chung WY: Platycodin D blocks breast cancer-induced bone destruction by inhibiting osteoclastogenesis and the growth of breast cancer cells. Cell Physiol Biochem 36: 1809-1820, 2015. 
25. Tang ZH, Li T, Gao HW, Sun W, Chen XP, Wang YT and Lu JJ: Platycodin D from Platycodonis Radix enhances the antiproliferative effects of doxorubicin on breast cancer MCF-7 and MDA-MB-231 cells. Chin Med 9: 16, 2014.

26. Chun J, Ha IJ and Kim YS: Antiproliferative and apoptotic activities of triterpenoid saponins from the roots of Platycodon grandiflorum and their structure-activity relationships. Planta Med 79: 639-645, 2013.

27. Riley T, Sontag E, Chen P and Levine A: Transcriptional control of human p53-regulated genes. Nat Rev Mol Cell Biol 9: 402-412, 2008.

28. Hao Q and Cho WC: Battle against cancer: An everlasting saga of p53. Int J Mol Sci 15: 22109-22127, 2014.

29. Muller PA and Vousden KH: p53 mutations in cancer. Nat Cell Biol 15: 2-8, 2013

30. Hollstein M, Sidransky D, Vogelstein B and Harris CC: p53 mutations in human cancers. Science 253: 49-53, 1991.

31. Freed-Pastor WA and Prives C: Mutant p53: One name, many proteins. Genes Dev 26: 1268-1286, 2012.

32. Rivlin N, Brosh R, Oren M and Rotter V: Mutations in the p53 tumor suppressor gene: Important milestones at the various steps of tumorigenesis. Genes Cancer 2: 466-474, 2011.
33. Kim BM, Kim DH, Park JH, Surh YJ and Na HK: Ginsenoside $\mathrm{Rg} 3$ inhibits constitutive activation of NF- $\mathrm{KB}$ signaling in human breast cancer (MDA-MB-231) cells: ERK and Akt as potential upstream targets. J Cancer Prev 19: 23-30, 2014.

34. Zhang Q, Zeng SX and Lu H: Targeting p53-MDM2-MDMX loop for cancer therapy. Subcell Biochem 85: 281-319, 2014

35. Pei D, Zhang Y and Zheng J: Regulation of p53: A collaboration between Mdm2 and Mdmx. Oncotarget 3: 228-235, 2012.

36. Qin JJ, Wang W, Voruganti S, Wang H, Zhang WD and Zhang R: Identification of a new class of natural product MDM2 inhibitor: In vitro and in vivo anti-breast cancer activities and target validation. Oncotarget 6: 2623-2640, 2015.

37. Olivier M, Eeles R, Hollstein M, Khan MA, Harris CC and Hainaut P: The IARC TP53 database: New online mutation analysis and recommendations to users. Hum Mutat 19: 607-614, 2002.

38. Katayose D, Gudas J, Nguyen H, Srivastava S, Cowan KH and Seth P: Cytotoxic effects of adenovirus-mediated wild-type p53 protein expression in normal and tumor mammary epithelial cells. Clin Cancer Res 1: 889-897, 1995.

39. Walerych D, Napoli M, Collavin L and Del Sal G: The rebel angel: Mutant p53 as the driving oncogene in breast cancer. Carcinogenesis 33: 2007-2017, 2012. 